\title{
Letramento funcional em saúde em adultos e idosos com disfagia
}

\author{
Functional health literacy in adults and elderly with dysphagia \\ Urssula Aparecida Santos Leal Ribeiro' (D), Laélia Cristina Caseiro Vicente² (i) Stela Maris Aguiar Lemos²
}

\begin{abstract}
RESUMO
Objetivo: Verificar a associação do letramento funcional em saúde de adultos e idosos com disfagia com os dados socioeconômicos, clínicos e de habilidade de deglutição, de acordo com o rastreio cognitivo. Métodos: Estudo observacional analítico transversal, com 49 participantes com disfagia de um ambulatório de reabilitação. Os procedimentos para coleta de dados foram análise dos prontuários, Critério de Classificação Econômica Brasil, Mini Exame do Estado Mental, Short Assessment of Health Literacy for Portuguese-speaking Adults, Protocolo Fonoaudiológico de Avaliação do Risco para Disfagia, Functional Oral Intake Scale e Índice de Desvantagem da Disfagia. Foram realizadas análises descritiva, bivariada e regressão logística múltipla. Resultados: A maioria dos participantes apresentou letramento funcional em saúde inadequado, doença neurológica, escore superior no rastreio cognitivo, disfagia leve e moderada e alimentava-se por via oral, com restrições. Houve associação na regressão logística múltipla do letramento funcional em saúde com a escolaridade e a doença de base para o grupo com escore superior, no rastreio cognitivo. Os participantes com maior escolaridade apresentaram 11,9 mais chances de ter letramento funcional em saúde adequado; os participantes neurológicos demonstraram 93,0 vezes mais chances de apresentar o letramento em saúde inadequado. Conclusão: Indivíduos com maior escolaridade e aqueles que não apresentavam doença neurológica demonstraram menos chance de ter letramento funcional em saúde inadequado.
\end{abstract}

Palavras-chave: Alfabetização em saúde; Transtornos de deglutição; Adulto; Idoso; Assistência ambulatorial

\begin{abstract}
Purpose: To verify the association between Functional Health Literacy in adults and elderly with dysphagia and socioeconomic, clinical, and swallowing ability data according to cognitive screening. Methods: Observational analytical cross-sectional study with 49 participants in a rehabilitation clinic. We analyzed medical records, Brazil Economic Classification Criteria, Mini Mental State Examination, Short Assessment of Health Literacy for Portuguese-speaking Adults, Speech Therapy Protocol for Dysphagia Risk Assessment, Functional Oral Intake Scale, and Dysphagia Handicap Index. Descriptive, bivariate, and multiple logistic regression analyzes were performed. Results: Most participants presented functional literacy in inadequate health, neurological disease, higher score on cognitive performance, mild and moderate dysphagia, and were fed orally with restrictions. There was an association in the multiple logistic regression of functional health literacy with schooling and underlying disease for the group with a higher score on cognitive performance, participants with higher education were 11.9 more likely to have adequate health literacy, neurological participants demonstrated 93.0 times more likely to have inadequate health literacy. Conclusion: Individuals with a higher level of education and those who did not have underlying neurological disease sowed less chance of having inadequate Functional Health Literacy.
\end{abstract}

Keywords: Health literacy; Deglutition disorders; Adult; Elderly; Ambulatory care

Trabalho realizado no Departamento de Fonoaudiologia - Universidade Federal de Minas Gerais - UFMG - Belo Horizonte (MG), Brasil.

${ }^{1}$ Residência Multiprofissional em Saúde, Área de Concentração Saúde do Idoso, Hospital das Clínicas, Universidade Federal de Minas Gerais - UFMG, Belo Horizonte (MG), Brasil.

${ }^{2}$ Departamento de Fonoaudiologia, Faculdade de Medicina, Universidade Federal de Minas Gerais - UFMG - Belo Horizonte (MG), Brasil.

Conflito de interesses: Não.

Contribuição dos autores: UASLR participou da coleta, análise e interpretação dos dados e redação do artigo; LCCV participou, na condição de coorientadora, da idealização do estudo, análise e interpretação dos dados e correção do manuscrito; SMAL participou, na condição de orientadora, da idealização do estudo, análise e interpretação dos dados e correção do manuscrito.

Financiamento: Nada a declarar.

Autor correspondente: Urssula Aparecida Santos Leal Ribeiro. E-mail: urssulaleal@gmail.com

Recebido: Maio 25, 2020; Aceito: Dezembro 08, 2020 


\section{INTRODUÇÃO}

O letramento funcional em saúde (LFS) é a capacidade de entender, interpretar e aplicar informações escritas, faladas ou numéricas, necessárias para compreender e transitar nos ambientes de cuidados de saúde ${ }^{(1)}$. Um baixo LFS pode dificultar a promoção e a educação em saúde e está associado aos comportamentos de risco, tais como redução do autocuidado, aumento de hospitalizações e custos ${ }^{(2)}$. Nesse contexto, considera-se importante não apenas a capacidade de leitura e escrita, mas o que o sujeito é capaz de fazer com as informações de saúde obtidas ${ }^{(1)}$. Acredita-se que uma pessoa com nível de LFS satisfatório transite melhor pelos ambientes de saúde, tendo, assim, melhores condições para o autocuidado e para o gerenciamento de condições de saúde ${ }^{(1,2)}$. Autores apontaram que o LFS pode ser uma ferramenta para a promoção da saúde, propiciando aos sujeitos condições para exercer controle sobre a saúde e melhorá-la ${ }^{(3)}$.

Os distúrbios de deglutição podem ser ocasionados por acometimentos neurológicos, estruturais, mecânicos, senis ou psicogênicos ${ }^{(4)}$. Consistem em qualquer dificuldade em transportar o bolo alimentar da cavidade oral até o estômago, sendo fator de risco para desnutrição, desidratação e podendo ocasionar pneumonia aspirativa ${ }^{(4)}$. $\mathrm{O}$ tratamento das disfagias orofaríngeas envolve adaptações, ações reabilitadoras e orientações que visam minimizar os riscos de uma deglutição ineficiente. Diante disso, acredita-se que o LFS adequado pode ser um facilitador no manejo clínico, tendo em vista que pode influenciar a percepção que o sujeito tem sobre sua condição de saúde e o desempenho em atividades de autocuidado.

Investigar o letramento funcional em saúde em pessoas com disfagia se faz importante, considerando que a assistência e adesão ao tratamento têm como eixo fundamental a participação do indivíduo. Desse modo o construto LFS não pode ser negligenciado pelos profissionais de saúde, incluindo o fonoaudiólogo envolvido nos processos terapêuticos e diagnósticos, que deve buscar a triangulação com fatores cognitivos, de escolaridade, socioeconômicos e gravidade da disfagia. Sendo assim, o objetivo do presente estudo foi verificar a associação do letramento funcional em saúde de adultos e idosos com disfagia aos dados socioeconômicos, clínicos e de habilidade de deglutição, de acordo com o rastreio cognitivo.

\section{MÉTODOS}

Trata-se de estudo observacional analítico transversal, com amostra não probabilística, composta por 49 adultos e idosos usuários de um ambulatório de reabilitação de disfagia de um hospital público. O estudo foi aprovado pelo comitê de ética em pesquisa da instituição, sob parecer número 3.006.459.

Todos os participantes foram informados sobre os objetivos e procedimentos da pesquisa, concordaram em participar e assinaram o Termo de Esclarecimento Livre e Esclarecido (TCLE).

Foram incluídos na pesquisa sujeitos com idade superior a 18 anos, que apresentavam disfagia orofaríngea e eram atendidos no Ambulatório de Fonoaudiologia do Hospital das Clínicas da Universidade Federal de Minas Gerais. Foram excluídos aqueles que não apresentaram condições neurológicas e/ou cognitivas para compreender os procedimentos propostos, sendo dependentes para as atividades de vida diária, de acordo com a Medida de Independência Funcional (MIF) ${ }^{(5)}$. Todos os participantes estavam em terapia fonoaudiológica para disfagia, no mínimo há um mês, no momento da coleta de dados. Os casos neurológicos foram doença de Parkinson, doença de Huntington, hemangioblastoma de fossa posterior, distrofia miotônica de Steinert, paralisia cerebral, atrofia muscular, traumatismo cranioencefálico, miastenia gravis, acidente vascular cerebral, distonia generalizada, esclerose múltipla, síndrome de Guillain-Barré e ataxia cerebelar. Já os casos não neurológicos foram disfagia pós-ventilação mecânica prolongada, pós-operatório tardio de tireoidectomia, disfagia secundária à cardiopatia, disfagia de condução devido à presença de divertículo e disfagia após trauma por arma branca. Todos os participantes eram capazes de realizar atividades motoras e cognitivas sem solicitação de ajuda a terceiros, no mínimo de acordo com o nível de dependência moderada da escala MIF. Sendo assim, o desempenho funcional dos participantes do estudo variou entre independência completa e dependência moderada, sem comprometer a funcionalidade para responder aos protocolos propostos ${ }^{(5)}$.

Os dados sociodemográficos de caracterização dos usuários (idade, escolaridade, local de residência e doença de base) foram coletados dos prontuários. A coleta dos demais procedimentos foi realizada no dia que o participante compareceu para terapia, em sala reservada, por meio de entrevista individual, com duração de, aproximadamente, 30 minutos. Os protocolos aplicados seguiram a ordem descrita a seguir. Para a escolaridade, consideraram-se os grupos de menor escolaridade (composto por indivíduos que cursaram ensino fundamental incompleto, ensino fundamental completo e ensino médio incompleto) e de maior escolaridade (composto por aqueles com ensino médio e superior).

O rastreio cognitivo foi mensurado por meio do Mini Exame do Estado Mental (MEEM), pois fornece informações sobre diferentes domínios cognitivos, agrupados nas categorias de orientação temporal, orientação espacial, atenção e cálculo, memória, linguagem e capacidade visoconstrutiva ${ }^{6)}$. Cabe ressaltar que, para a análise de associação, os participantes foram distribuídos segundo o rastreio cognitivo. O escore do MEEM foi utilizado para distribuir os participantes nos grupos de escore inferior (G1) e escore superior (G2). Foram considerados pontos de corte segundo a escolaridade, sendo que, para obter escore superior, os participantes deveriam apresentar os seguintes escores: analfabetos, escore superior a 20; escolaridade de um a quatro anos, escore superior a 25; escolaridade de cinco a oito anos, escore superior a 26,5; escolaridade entre nove e 11 anos, escore superior a 28; escolaridade acima de 11 anos, escore 29(6).

Para conhecimento das condições socioeconômicas, foi aplicado o Critério de Classificação Econômica Brasil (CCEB), sob a forma de entrevista com o participante ${ }^{(7)}$. O instrumento leva em consideração a escolaridade do chefe da família e utiliza o levantamento de características domiciliares referentes à presença e quantidade de itens domésticos.

O letramento funcional em saúde foi pesquisado por meio do protocolo Short Assessment of Health Literacy for Portuguesespeaking Adults (SAHLPA) ${ }^{(8)}$. É um instrumento adaptado e validado por pesquisadores brasileiros que o disponibilizam para utilização mediante solicitação dos interessados e foi autorizado para uso nesta pesquisa. O instrumento avalia as habilidades de leitura, pronúncia e compreensão de 18 termos médicos comuns, em que cada item correto recebe um ponto e o escore total é obtido pela soma dos itens, variando de 0 a 18. 
Os itens são considerados corretos apenas quando a pronúncia e a associação são realizadas acertadamente. Escores no intervalo de 0 a 14 sugerem alfabetismo em saúde inadequado e de 15 a 18 , alfabetismo em saúde adequado ${ }^{(8)}$.

A investigação clínica da deglutição foi realizada por meio do Protocolo Fonoaudiológico de Avaliação do Risco Para Disfagia (PARD), constituído por testes de deglutição de água e de alimento pastoso. A partir dessa análise, classifica-se o grau de habilidade da deglutição em sete níveis, que vão de deglutição normal (nível 1) até disfagia orofaríngea grave (nível 7) ${ }^{(9)}$.

Para caracterizar a via de alimentação dos participantes, no momento da coleta, foi utilizada a Functional Oral Intake Scale (FOIS) ${ }^{(10)}$. É uma escala clínica, composta por sete níveis: 1 - nada por via oral; 2 - dependente de via alternativa e mínima via oral de algum alimento ou líquido; 3 - dependente de via alternativa com consistente via oral de alimento ou líquido; 4- via oral total de uma única consistência; 5- via oral total com múltiplas consistências, porém, com necessidade de preparo especial ou compensações; 6- via oral total com múltiplas consistências, sem necessidade de preparo especial ou compensações, porém, com restrições alimentares; 7- via oral total sem restrições.

A limitação de atividade e restrição à participação em disfagia foi verificada por meio do Índice de Desvantagem da Disfagia (IDD) ${ }^{(11)}$. Composto pelos domínios físico, funcional e emocional, o instrumento contém 25 questões, com as possibilidades de respostas nunca ( 0 pontos), às vezes ( 2 pontos) e sempre (4 pontos), sendo o escore em cada domínio calculado pela soma das pontuações das questões envolvidas. O protocolo apresenta, ainda, escala Likert, com valores de 0 a 7, por meio da qual o participante realiza autoavaliação da dificuldade relacionada à deglutição. Quanto maior o escore, pior a limitação de atividade e restrição à participação em disfagia.

A variável resposta foi o letramento funcional em saúde (LFS -SALPHA) e as variáveis explicativas foram idade, gênero, escolaridade, classe econômica, gravidade da disfagia, restrição à participação em deglutição e via de alimentação. Para análise de muitas variáveis explicativas, os dados obtidos foram agrupados conforme exposto a seguir.

Quanto à gravidade da deglutição, os níveis do PARD foram redistribuídos em três categorias: alteração leve (deglutição funcional e disfagia leve), moderada (disfagia leve/moderada e disfagia moderada) e grave (disfagia moderada/grave e disfagia grave). Já na escala FOIS, os agrupamentos foram: uso de via alternativa (FOIS 1,2 e 3), via oral com uma consistência (FOIS 4), via oral com restrição (FOIS 5 e 6) e via oral sem restrições (FOIS 7).

A análise descritiva constou da distribuição de frequência para as variáveis categóricas e das medidas de tendência central e de dispersão para as variáveis contínuas. A análise bivariada foi realizada por meio dos testes de Qui-Quadrado e Exato de Fisher, considerando 5\% de significância. Adotou-se o método de seleção de Backward para associar o letramento funcional em saúde com as variáveis sociodemográficas e clínicas. As variáveis que tiveram associação em nível de $20 \%(\mathrm{p} \leq 0,20)$ com as variáveis resposta foram consideradas para o modelo de regressão logística. Posteriormente no modelo de regressão logística múltipla, foram consideradas as variáveis com associação significativa em nível de 5\% $(\mathrm{p} \leq 0,05)$. A magnitude das associações foi avaliada pela
Odds Ratio (OR) e seus respectivos intervalos de confiança a $95 \%$. As analises foram realizadas por meio do programa IBM SPSS Statistics, versão 24.

\section{RESULTADOS}

A maioria dos participantes apresentou LFS inadequado $(53,1 \%)$ e escore superior no rastreio cognitivo $(57,1 \%)$. A idade variou de 24 a 88 anos, com média de 58,4 anos (desvio padrão $\pm 16,8$ anos), sendo os idosos mais frequentes $(51,0 \%)$. Prevaleceram indivíduos do gênero feminino $(67,3 \%)$, residentes na cidade de Belo Horizonte/Minas Gerais $(63,2 \%)$ e com doenças neurológicas $(73,5 \%)$. Quanto à escolaridade, não houve predominância entre as categorias. A classe econômica mais observada foi a $\mathrm{C}$ $(63,3 \%)$ (Tabela 1$)$.

Quanto à caracterização da deglutição, a gravidade da disfagia variou, predominantemente, entre leve $(51,1 \%)$ e moderada $(44,9 \%)$, sendo que a maioria se alimentava por via oral com mais de uma consistência, todavia com restrições $(75,5 \%)$. Mais de um terço da amostra se autoavaliou com problema moderado de deglutição. A limitação de atividade e restrição à participação em disfagia apresentou total médio de 38,7 pontos, em um mínimo de 0 e total de 100 pontos, sendo que, quanto mais próximo do total, maior a limitação de atividade e restrição à participação em disfagia (Tabela 1). Na pontuação por domínios, o domínio físico médio foi de 14,4 pontos em um máximo de 36 , o domínio funcional médio, de 16 pontos em um máximo de 36 e o domínio emocional médio, de 10 pontos, sendo o máximo de 28 pontos (Figura 1). Quanto mais próximo da pontuação total em cada um dos domínios, maior o impacto causado pela disfagia orofaríngea. O rastreio cognitivo apresentou média de 23,6 em escore máximo de 30, com mínimo de 14,0 e mediana de 24 (desvio padrão $\pm 4,2$ ).

$\mathrm{Na}$ análise de associação do LFS segundo os dados sociodemográficos, clínicos e funcionais, de acordo com o rastreio cognitivo, não houve associação com as variáveis pesquisadas (Tabela 2). As associações do LFS com as variáveis sociodemográficas, clínicas e funcionais, independente do rastreio cognitivo, não demonstraram associação com significância entre as variáveis (Tabela 3 ).

Foi realizada análise multivariada por regressão logística múltipla hierarquizada dos fatores idade, gênero, escolaridade, doença de base, classe econômica, gravidade da deglutição, via de alimentação e restrição à participação em deglutição com o LFS. Não houve associação estatisticamente significativa das variáveis sociodemográficas, clínicas e funcionais com o LFS para o grupo de escore inferior. Entretanto, os achados demonstraram que, no modelo final da regressão logística múltipla, para o grupo de escore superior, se mantiveram associadas ao LFS a escolaridade, $\mathrm{p} \leq 0,04$, e a doença de base, $\mathrm{p} \leq 0,03$ (Tabela 4). Desse modo, verificou-se que as chances dos participantes com maior escolaridade terem letramento funcional em saúde adequado foram $11,9 \%$ vezes maiores do que os que possuíam menor escolaridade. Os participantes que apresentaram doença de base de etiologia neurológica demonstraram 93,0\% vezes mais chances de apresentar o letramento em saúde inadequado, quando comparados àqueles com doença de base não neurológica. 
Tabela 1. Caracterização da amostra segundo dados sociodemográficos e clínicos

\begin{tabular}{|c|c|c|c|}
\hline & & $\mathbf{n}$ & $\%$ \\
\hline \multirow[t]{2}{*}{ Ciclo de vida } & Adulto & 24 & 49 \\
\hline & Idoso & 25 & 51 \\
\hline \multirow[t]{2}{*}{ Gênero } & Masculino & 16 & 32,6 \\
\hline & Feminino & 33 & 67,3 \\
\hline \multirow[t]{2}{*}{ Escolaridade } & Menor (ensino fundamental incompleto a ensino médio incompleto) & 25 & 51 \\
\hline & Maior (ensino médio e superior) & 24 & 49 \\
\hline \multirow[t]{3}{*}{ Residência } & Belo Horizonte & 31 & 63,2 \\
\hline & Região metropolitana & 7 & 14,2 \\
\hline & Interior & 11 & 22,4 \\
\hline \multirow[t]{7}{*}{ CCEB } & A1 & 0 & 0 \\
\hline & $\mathrm{A} 2$ & 0 & 0 \\
\hline & B1 & 1 & 2 \\
\hline & B2 & 9 & 18,4 \\
\hline & $\mathrm{C} 1$ & 17 & 34,7 \\
\hline & $\mathrm{C} 2$ & 14 & 28,6 \\
\hline & $\mathrm{D}$ e $\mathrm{E}$ & 8 & 16,3 \\
\hline \multirow[t]{2}{*}{ Letramento Funcional em Saúde } & Adequado & 23 & 46,9 \\
\hline & Inadequado & 26 & 53,1 \\
\hline \multirow[t]{2}{*}{ Doença de base } & Neurológico & 36 & 73,5 \\
\hline & Não neurológico & 13 & 26,5 \\
\hline \multirow[t]{2}{*}{ Mini Exame do Estado Mental } & Escore superior & 28 & 57,1 \\
\hline & Escore inferior & 21 & 42,9 \\
\hline \multirow[t]{3}{*}{ PARD } & Alteração leve & 25 & 51,1 \\
\hline & Alteração moderada & 22 & 44,9 \\
\hline & Alteração grave & 2 & 4 \\
\hline \multirow[t]{4}{*}{ FOIS } & Uso de via alternativa & 1 & 2 \\
\hline & Via oral uma consistência & 3 & 6,1 \\
\hline & Via oral com restrição & 37 & 75,5 \\
\hline & Via oral sem restrições & 8 & 16,4 \\
\hline \multirow{4}{*}{$\begin{array}{l}\text { Autoavaliação da deglutição } \\
\text { segundo limitação de atividade e } \\
\text { restrição à participação de disfagia } \\
\text { - (IDD) }\end{array}$} & Normal & 1 & 2 \\
\hline & Problema leve & 10 & 20,4 \\
\hline & Problema moderado & 23 & 46,9 \\
\hline & Problema grave & 15 & 30,7 \\
\hline
\end{tabular}

Legenda: $\mathrm{n}$ = número de sujeitos; \% = percentual; CCEB = Critério de Classificação Econômica Brasil; A1, A2, B1, B2, C1, C2, D, E = classes econômicas; PARD = Protocolo da Avaliação do Risco de Disfagia; FOIS = Escala Funcional de Ingestão por Via Oral (Functional Oral Intake Scale); IDD = Índice de Desvantagem em Deglutição

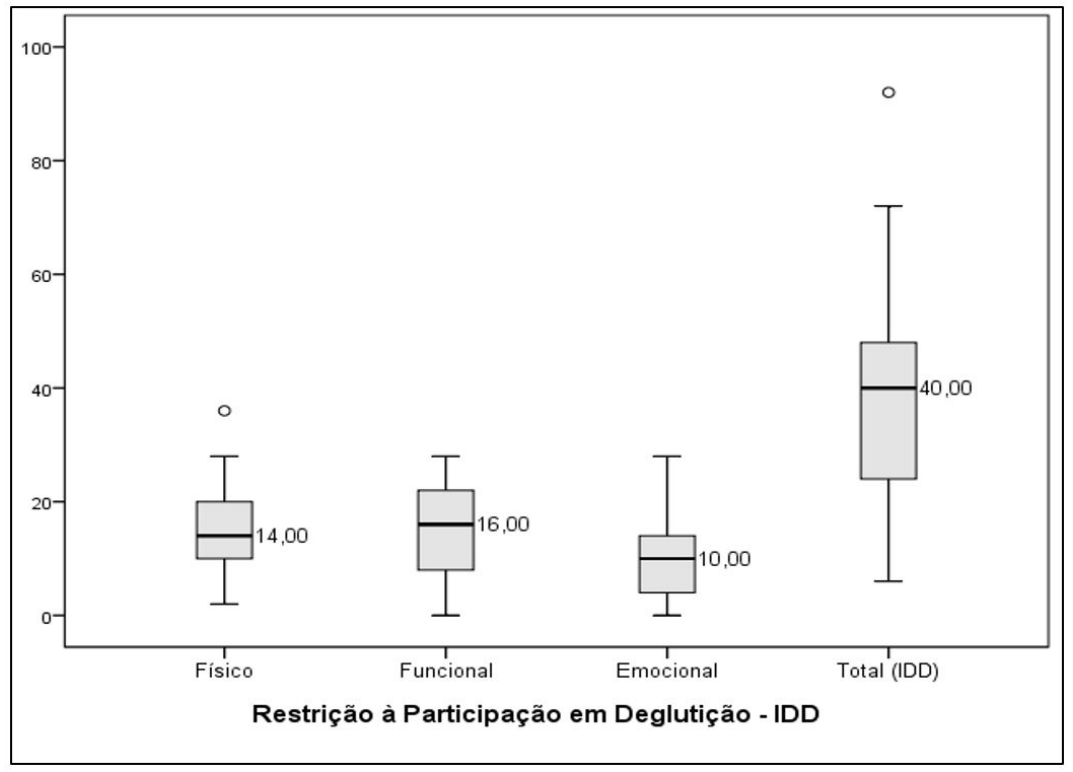

Figura 1. Caracterização da limitação de atividade e restrição à participação em disfagia segundo o Índice de Desvantagem em Deglutição Legenda: IDD = Índice de Desvantagem em Deglutição 


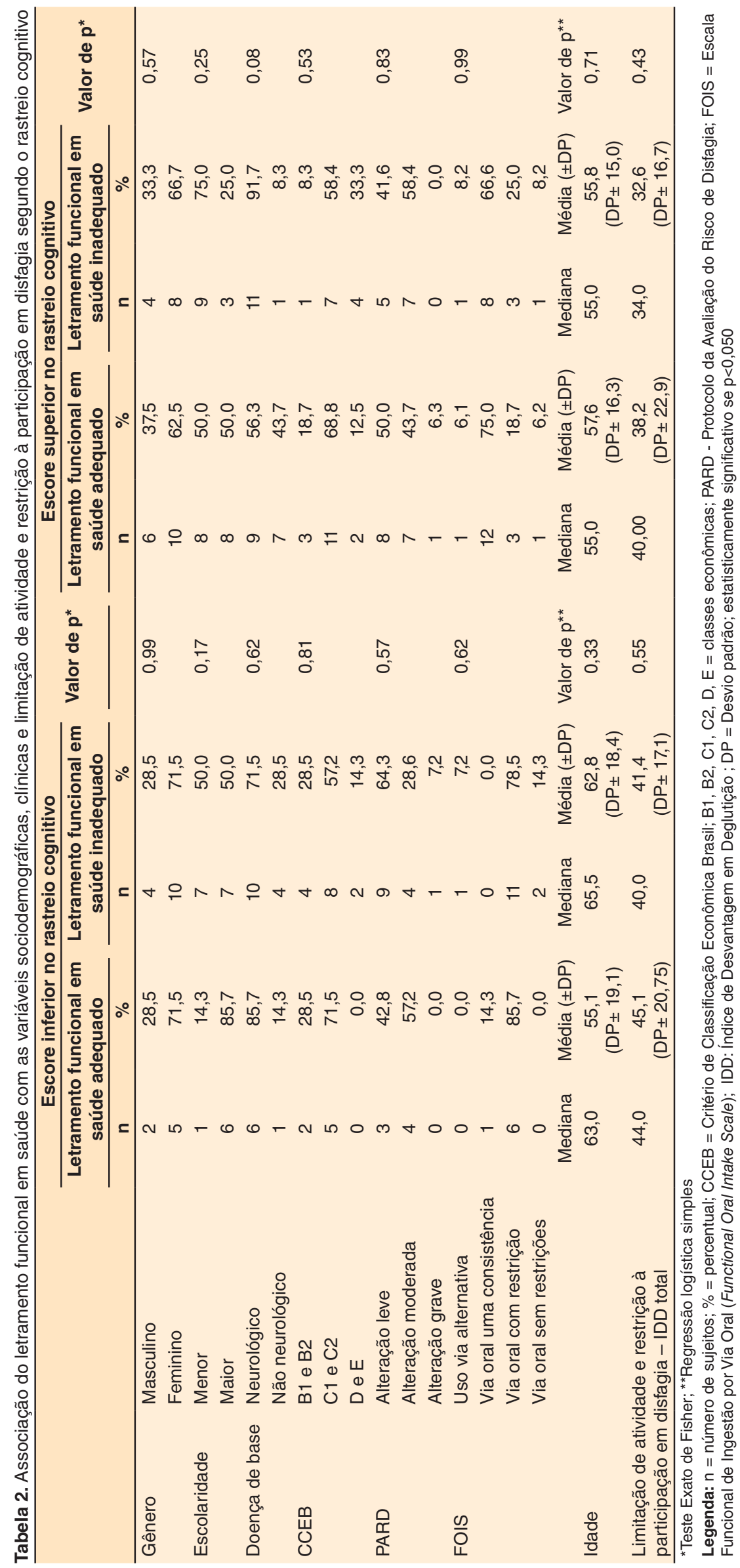


Tabela 3. Associação do letramento funcional em saúde com as variáveis sociodemográficas, clínicas e limitação de atividade e restrição à participação em disfagia independente do rastreio cognitivo

\begin{tabular}{|c|c|c|c|c|c|c|}
\hline & \multicolumn{4}{|c|}{ Letramento funcional em saúde } & \multirow{3}{*}{ Valor de $p^{\star}$} \\
\hline & & \multicolumn{2}{|c|}{ Adequado } & \multicolumn{2}{|c|}{ Inadequado } & \\
\hline & & $\mathbf{n}$ & $\%$ & $\mathrm{n}$ & $\%$ & \\
\hline \multirow[t]{2}{*}{ Gênero } & Masculino & 8 & 34,78 & 8 & 30,77 & 0,999 \\
\hline & Feminino & 15 & 65,22 & 18 & 69,23 & \\
\hline \multirow[t]{2}{*}{ Escolaridade } & Menor escolaridade & 9 & 39,13 & 16 & 61,54 & 0,156 \\
\hline & Maior escolaridade & 14 & 60,87 & 10 & 38,46 & \\
\hline \multirow[t]{2}{*}{ Doença de base } & Neurológico & 15 & 65,22 & 21 & 80,77 & 0,332 \\
\hline & Não neurológico & 8 & 34,78 & 5 & 19,23 & \\
\hline \multirow[t]{3}{*}{ CCEB } & B1 e B2 & 5 & 21,74 & 5 & 19,23 & 0,459 \\
\hline & $\mathrm{C} 1$ e $\mathrm{C} 2$ & 16 & 69,57 & 15 & 57,69 & \\
\hline & $\mathrm{De} E$ & 2 & 8,70 & 6 & 23,08 & \\
\hline \multirow[t]{3}{*}{ PARD } & $\begin{array}{l}\text { Alteração leve (deglutição } \\
\text { funcional + disfagia leve) }\end{array}$ & 11 & 47,83 & 14 & 53,85 & 0,885 \\
\hline & $\begin{array}{l}\text { Alteração moderada (disfagia } \\
\text { leve/moderada + disfagia } \\
\text { moderada) }\end{array}$ & 11 & 47,83 & 11 & 42,31 & \\
\hline & $\begin{array}{l}\text { Alteração grave (disfagia } \\
\text { moderada/grave + disfagia grave) }\end{array}$ & 1 & 4,35 & 1 & 3,85 & \\
\hline \multirow[t]{5}{*}{ FOIS } & $\begin{array}{l}\text { Restrição de alimentos por via } \\
\text { oral com uso de via alternativa }\end{array}$ & 0 & 0,00 & 1 & 3,85 & 0,822 \\
\hline & Via oral uma consistência & 2 & 8,70 & 1 & 3,85 & \\
\hline & $\begin{array}{l}\text { Via oral mais de uma } \\
\text { consistência, mas com preparo } \\
\text { especial }\end{array}$ & 18 & 78,26 & 19 & 73,08 & \\
\hline & Via oral sem restrições & 3 & 13,04 & 5 & 19,23 & \\
\hline & & Mediana & Média ( $(\mathrm{DP})$ & Mediana & Média $( \pm D P)$ & Valor de $\mathrm{p}^{\star \star}$ \\
\hline \multicolumn{2}{|l|}{ Idade } & 60,0 & $56,9(\mathrm{DP} \pm 16,8)$ & 59,0 & $59,7(\mathrm{DP} \pm 17,0)$ & 0,542 \\
\hline \multicolumn{2}{|c|}{$\begin{array}{l}\text { Limitação de atividade e restrição à participação em } \\
\text { disfagia segundo o IDD total }\end{array}$} & 44,00 & $\begin{array}{c}40,35 \\
(\mathrm{DP} \pm 22,10)\end{array}$ & 39,00 & $\begin{array}{c}37,38 \\
(\mathrm{DP} \pm 17,17) \\
\end{array}$ & 0,583 \\
\hline
\end{tabular}

*Teste Exato de Fisher ou Qui-Quadrado de Pearson; **Regressão logística simples

Legenda: $\mathrm{n}=$ número de sujeitos; $\%=$ percentual; $\mathrm{CCEB}=$ Critério de Classificação Econômica Brasil; $\mathrm{B} 1, \mathrm{~B} 2, \mathrm{C} 1, \mathrm{C} 2, \mathrm{D}, \mathrm{E}=\mathrm{classes}$ econômicas; PARD = Protocolo da Avaliação do Risco de Disfagia; FOIS = Escala Funcional de Ingestão por Via Oral (Functional Oral Intake Scale); IDD = Índice de desvantagem em deglutição; $\mathrm{DP}=$ Desvio padrão; estatisticamente significativo se $\mathrm{p}<0,050$

Tabela 4. Análise por regressão logística do letramento funcional em saúde com escolaridade e doença de base

\begin{tabular}{|c|c|c|c|c|c|c|c|c|c|c|c|c|c|}
\hline \multirow[t]{3}{*}{ Rastreio } & \multicolumn{5}{|c|}{ Escore inferior } & \multicolumn{4}{|c|}{ Escore superior } & \multicolumn{4}{|c|}{ Independente do escore } \\
\hline & & \multirow{2}{*}{ OR } & \multicolumn{2}{|c|}{$\begin{array}{c}\text { Intervalo de } \\
\text { confiança de } 95 \% \\
\text { para OR }\end{array}$} & \multirow{2}{*}{$\begin{array}{l}\text { Valor } \\
\text { de } p^{*}\end{array}$} & \multirow{2}{*}{ OR } & \multicolumn{2}{|c|}{$\begin{array}{l}\text { Intervalo de } \\
\text { confiança de } \\
95 \% \text { para OR }\end{array}$} & \multirow{2}{*}{$\begin{array}{l}\text { Valor } \\
\text { de } p^{*}\end{array}$} & \multirow{2}{*}{ OR } & \multicolumn{2}{|c|}{$\begin{array}{c}\text { Intervalo de } \\
\text { confiança de } 95 \% \\
\text { para OR } \\
\end{array}$} & \multirow{2}{*}{$\begin{array}{l}\text { Valor } \\
\text { de } p^{*}\end{array}$} \\
\hline & & & $\begin{array}{l}\text { Limite } \\
\text { inferior }\end{array}$ & $\begin{array}{l}\text { Limite } \\
\text { superior }\end{array}$ & & & $\begin{array}{l}\text { Limite } \\
\text { inferior }\end{array}$ & $\begin{array}{l}\text { Limite } \\
\text { superior }\end{array}$ & & & $\begin{array}{l}\text { Limite } \\
\text { inferior }\end{array}$ & $\begin{array}{l}\text { Limite } \\
\text { superior }\end{array}$ & \\
\hline Escolaridade & Menor & - & 1 & - & - & 1 & - & - & - & 1 & - & - & - \\
\hline \multirow[t]{2}{*}{ Doença de base } & Neurológico & - & 1 & - & - & 1 & - & - & - & 1 & - & - & - \\
\hline & Não neurológico & 4,01 & 0,31 & 52,12 & 0,28 & 0,07 & 0,00 & 0,82 & 0,03 & 0,31 & 0,07 & 1,36 & 0,12 \\
\hline
\end{tabular}

${ }^{*}$ Regressão logística múltipla com método de seleção de Backward; Variáveis inseridas no modelo inicial: idade, gênero, escolaridade, doença de base, Critério de Classificação Econômica Brasil; Protocolo da Avaliação do Risco de Disfagia; Escala Funcional de Ingestão por Via Oral (Functional Oral Intake Scale);e Restrição à participação em deglutição

Legenda: OR = Odds Ratio; LFS = Letramento Funcional em Saúde

\section{DISCUSSÃO}

A maioria dos participantes deste estudo apresentou LFS inadequado. Pesquisas que investigaram o LFS em amostras de atenção primária à saúde e componente especializado (ambulatório), que apresentavam diabetes e doença renal crônica em tratamento pré-dialítico, observaram LFS inadequado em proporções maiores que as do presente estudo ${ }^{(12,13)}$. Para os indivíduos com diabetes, mais de dois terços apresentaram LFS inadequado e todos aqueles com doença renal crônica apresentaram desempenho inadequado ${ }^{(12,13)}$. O grau de escolaridade dos sujeitos do presente estudo era maior do que o dos estudos citados e, embora a escolaridade não garanta o nível adequado de LFS, a literatura aponta que indivíduos com maior escolaridade podem ter melhor desempenho de LFS, o que independe de 
sua área de formação. Vale ressaltar, também, que diferente do instrumento utilizado neste estudo, que verifica a habilidade de leitura, pronúncia e compreensão por meio de 18 termos médicos comuns, o instrumento utilizado nas pesquisas com diabéticos e com sujeitos com doença renal crônica foi o Short Test of Funcional Literacy in Adults (S-TOFHLA), composto por 36 itens divididos em dois subtestes que avaliavam as habilidades de compreensão textual por meio de frases com lacunas a serem preenchidas pelo participante em determinado tempo e de numeramento ${ }^{(14)}$.

Quanto à prevalência de idosos, segundo dados do Instituto Brasileiro de Geografia e Estatística (IBGE), é crescente a população de idosos e a expectativa de vida da população acima de $60 \operatorname{anos}^{(15)}$. Ao buscar na literatura estudos que investigaram o LFS nos diferentes ciclos de vida, em estudo realizado com idosos na Região Sul do Brasil, por meio do S-TOFHLA, observouse resultado inadequado em mais de um terço da amostra e, também, que o LFS pode diminuir com o avançar da idade ${ }^{(16)}$. Ao se considerarem os adultos, o LFS inadequado, na maioria da amostra, também foi encontrado em estudos realizados na atenção primária à saúde na Região Sudeste, sendo utilizado o mesmo instrumento deste estudo, e na Região Norte, por meio do S-TOFHLA ${ }^{(17,18)}$. Não foi observada associação da variável idade com o LFS neste estudo e uma possível justificativa foi o número reduzido de participantes.

Assim como no presente estudo, outras pesquisas ${ }^{(12-14,16-18)}$ que investigaram o LFS tiveram prevalência do gênero feminino em suas amostras. Dados do último censo do IBGE-2010 apontaram maior proporção de mulheres, em relação a homens. Além disso, quando se busca identificar os fatores associados às diferenças pela procura por serviços de saúde entre homens e mulheres, verifica-se que ser do gênero feminino pode ser um fator preditor de maior busca por assistência ${ }^{(15,19)}$.

Ao pesquisar estudos realizados em contexto de ambulatórios de reabilitação, observou-se que o LFS também foi tema de interesse em outras áreas ou campos de estudo ${ }^{(20,21)}$. Em estudo da área de Fisioterapia, realizado na Turquia, com objetivo de determinar o LFS em 423 indivíduos com distúrbios osteomusculares crônicos, observou-se que mais de dois a cada dez pacientes apresentavam LFS inadequado ${ }^{(20)}$. A pesquisa utilizou dois instrumentos para triagem do LFS e constatou que havia boa reprodutibilidade e concordância entre os instrumentos. O primeiro instrumento avaliava a capacidade de leitura e pronúncia de 66 termos médicos para partes do corpo, que poderiam ser comuns ou leigos, para classificar o LFS em quatro níveis. O segundo apurava habilidades de alfabetização e numeração por meio de leitura de rótulo nutricional com seis questões e, de acordo com a quantidade de acertos, classificava o LFS em adequado ou inadequado. Já em estudo norte-americano da área de Terapia Ocupacional, que investigou se idosos com baixa visão apresentavam menor LFS, constatou-se, por meio do S-TOFHLA, que a baixa acuidade visual estava correlacionada com menores escores de LFS ${ }^{(21)}$. Em serviços ambulatoriais de reabilitação, nota-se sistematicidade dos atendimentos, com acesso constante aos serviços de saúde, além de haver contato direto entre o usuário e profissional de saúde, fatores que podem contribuir para melhor desempenho de LFS.

Na prática clínica fonoaudiológica em disfagia, há grande quantidade de informações a serem entendidas, aplicadas e revisadas pelos pacientes, sendo que, constantemente, os elementos são transmitidos por escrito, a fim de auxiliar na gestão de condições de saúde. Nesse contexto, os níveis comunicativo e crítico do LFS devem ser considerados para eficácia terapêutica. Em estudo de caso realizado na África do Sul, com sujeito de 43 anos de idade que apresentava disfagia orofaríngea e baixo nível de LFS, foram utilizadas estratégias de alta tecnologia, como gravações de áudio na forma de $\mathrm{CD} /$ cassete, contato telefônico e mensagem de texto, para maximizar o LFS e a recuperação de informações clínicas, sendo observado maior entendimento sobre o processo terapêutico e maior motivação para realizar as tarefas envolvidas no autocuidado para a reabilitação em domicílio $^{(22)}$. Dess forma, o fonoaudiólogo deve considerar o LFS do indivíduo para promover estratégias individualizadas que garantam a compreensão e a adesão dos procedimentos indicados na reabilitação da disfagia.

Vale ressaltar, ainda, que, embora a maioria da amostra deste estudo tenha apresentado via oral com restrições, mas com ingestão de múltiplas consistências, quase metade dos participantes se autoavaliou com problema moderado de deglutição. Assim, o fonoaudiólogo deve ficar atento ao nível de LFS do paciente sob seu cuidado. O adequado LFS pode diminuir as limitações de atividade e restrição à participação em disfagia, uma vez que pode favorecer a compreensão dos indivíduos para as estratégias terapêuticas que facilitam a deglutição eficiente e segura.

Os dados iniciais sobre LFS e disfagia precisam ser aprimorados, uma vez que são escassos os estudos que abordam a inter-relação entre essas variáveis. Na terapia fonoaudiológica para disfagia, há grande quantidade de informações a serem processadas pelo sujeito para o gerenciamento da condição de saúde, além do ambiente terapêutico. Assim, se faz relevante compreender as possíveis influências do LFS no processo de autocuidado e gerenciamento da saúde do indivíduo com disfagia. As limitações de atividade e restrição à participação em disfagia estão aportadas na estruturação do modelo biopsicossocial da Organização Mundial da Saúde e, em consequência, da Classificação Internacional de Funcionalidade, Incapacidade e Saúde (CIF), como norteadoras do cuidado em saúde. Desse modo, ao examinar a disfagia na perspectiva da funcionalidade humana, foi considerada a necessidade de mudança de uma abordagem baseada na doença e suas consequências para uma perspectiva ancorada na funcionalidade como um componente da saúde ${ }^{(23)}$ e no entendimento de que as situações de saúde são diversas e devem ser tratadas de forma distinta, segundo as limitações de atividades e restrições a participação de cada sujeito $^{(24,25)}$. Nesse contexto, fica claro que pacientes com graus similares de disfagia podem apresentar limitações distintas de atividade e restrição à participação em disfagia.

Permaneceram no modelo final da análise por regressão logística múltipla as variáveis escolaridade e doença de base. Para o grupo de escore superior no rastreio cognitivo, verificouse que indivíduos com maior escolaridade demonstraram menos chance de ter LFS inadequado, assim como aqueles que não apresentaram doença de base de etiologia neurológica.

Estudo apontou que o maior nível de escolaridade pode ser responsável por melhor desempenho de LFS e que as habilidades de leitura e escrita reduzidas podem limitar o acesso a informações de saúde ${ }^{(13)}$. Em pesquisa realizada com adultos usuários da atenção primária, na Região Sudeste do Brasil, os resultados demonstraram que a escolaridade se manteve associada ao LFS. Verificou-se que os adultos com ensino fundamental e médio apresentavam mais chances de ter o LFS inadequado, quando comparados àqueles com ensino superior ${ }^{(17)}$. Embora tenha havido associação entre a 
escolaridade e o LFS, autores apontaram que esse não é o único condicionante que influencia ou garante o nível de habilidade no LFS e que deve-se considerar outras dimensões do fenômeno, como, por exemplo, o acesso à rede de saúde e a qualidade da comunicação entre profissionais de saúde e usuários ${ }^{(14,26)}$. Nesse contexto, profissionais de saúde devem estar atentos ao fornecer informações de saúde, buscando se certificar, sempre, do grau de compreensão da informação fornecida. Em relação à doença de base, verificaram-se maiores chances de LFS inadequado nos sujeitos com disfagia, cuja etiologia da doença de base era neurológica. Patologias neurológicas podem exercer influência sobre habilidades motoras e cognitivas, apresentando caráter progressivo/degenerativo, ou não. Embora não tenha sido objeto do estudo analisar a doença neurológica e habilidades cognitivas como mediadoras do LFS, o uso do rastreio cognitivo como critério de inclusão e separação dos grupos permitiu alcance do objetivo do estudo e utilização dos instrumentos propostos. Ainda em relação a doença de base, estudo de coorte norte-americano realizado com 221 indivíduos atendidos por serviço de neurologia ambulatorial identificou que mais de um quarto dos participantes apresentou LFS baixo, sendo que aqueles com maior duração da doença neurológica estavam mais propensos a menores níveis de LFS, e quase um terço da amostra não foi capaz de nomear qualquer medicamento que utilizava $^{(27)}$. Uma vez que é frequente o público com doenças neurológicas para o atendimento fonoaudiológicos em disfagia, se faz necessário que o profissional seja sensível aos aspectos de LFS, escolaridade, acesso aos serviços de saúde, comunicação e suporte sociofamiliar, ao transmitir informações e orientações de saúde, a fim de garantir maior efetividade das ações no processo terapêutico.

Contudo, para o grupo que apresentou escore inferior no rastreio cognitivo, não houve associação com significância estatística entre as variáveis escolaridade e doença de base. Ao se comparar os grupos de escore superior e escore inferior, verificou-se maior ocorrência de LFS inadequado entre os sujeitos com escore inferior. O LFS representa a capacidade cognitiva de entender, interpretar e aplicar informações sobre a saúde e, nesse sentido, o rastreio cognitivo pode influenciar o processo de obtenção, processamento e interpretação da informação básica em saúde ${ }^{(1)}$. Estudo apontou que habilidades cognitivas e sociais podem ser determinantes na motivação de indivíduos para acesso, compreensão e utilização das informações de saúde ${ }^{(28)}$. Em estudo norte-americano, realizado com objetivo de investigar a correlação entre alfabetização em saúde, idade e habilidades cognitivas de idosos, observou-se que as habilidades de LFS se modificavam com o envelhecimento cognitivo, reforçando a necessidade de assegurar que tarefas de autocuidado em saúde para idosos tenham demandas cognitivas e de alfabetização apropriadas ao desempenho individual ${ }^{(29)}$. Nesse contexto, vale ressaltar o fato de que indivíduos idosos ou com comprometimento da cognição podem necessitar do auxílio de um cuidador para transitar nos ambientes de cuidado em saúde. Sendo assim, para maior efetividade das ações de saúde com esse público, pode se fazer relevante conhecer o nível de LFS do sujeito que exerce a função de cuidador.

Como limitações deste estudo, encontram-se o tamanho e o delineamento da amostra, fatores que não permitiram generalizações. As doenças de base encontradas foram diversificadas, não possibilitando estudo das condições de LFS específicas para cada uma delas. Além disso, o percurso terapêutico dos pacientes não foi controlado e, portanto, a influência do processo terapêutico no LFS não foi mensurada. Todavia, este é o primeiro estudo com a temática de LFS em disfagia, tendo realizado a análise em contexto de um ambulatório de reabilitação e utilizado instrumentos difundidos na área de disfagia e para avaliação do LFS. Para a melhor compreensão sobre o LFS e a disfagia, sugerem-se estudos com grupos mais específicos quanto aos processos funcionais e doenças de base, que analisem a relação entre LFS e a reabilitação do transtorno da deglutição, no que se refere à adesão e compreensão das estratégias terapêuticas e investiguem a influência do processo terapêutico no desempenho de LFS.

\section{CONCLUSÃO}

Não houve associação do letramento funcional em saúde com as variáveis sociodemográficas, clínicas e funcionais para o grupo de escore inferior, no rastreio cognitivo. Todavia, houve associação com as variáveis escolaridade e doença de base para o grupo de escore superior, no rastreio cognitivo. Desse modo, concluiu-se que indivíduos com maior escolaridade e aqueles que não apresentavam doença de base de etiologia neurológica demonstraram menos chance de ter LFS inadequado.

\section{REFERÊNCIAS}

1. Rocha PC, Lemos SMA. Conceptual aspects and factors associated with Functional Health Literacy: a literary review. CEFAC. 2016;18(1):21425. http://dx.doi.org/10.1590/1982-021620161819615.

2. WHO: World Health Organization. Regional Office for Europe. Health literacy: the solid facts [Internet]. 2013 [citado em 2020 Maio 25]. Disponível em: htttp://euro. who.int/_data/assets/pdf file/0008/190655/ e96854.pdf

3. Martins NFF, Abreu DPG, Silva BT, Bandeira EO, Lima JP, Mendes JM. Functional literacy on health of elderly people in a family health unit. Rev Enferm Cent Oeste Min. 2019;9:e2937. http://dx.doi. org/10.19175/recom.v9i0.2937.

4. Silva LML, Lima CR, Cunha DA, Orange LG. Dysphagia and its relation with nutritional status and calorie/protein intake in the elderly. Rev CEFAC. 2019;21(3):e15618. http://dx.doi.org/10.1590/1982$0216 / 201921315618$.

5. Riberto M, Miyazaki MH, Jorge Filho D, Sakamoto H, Battistella LR. Reprodutibilidade da versão brasileira da medida de independência funcional. Acta Fisiátrica. 2001;8(1):45-52. http://dx.doi.org/10.5935/01047795.20010002 .

6. Brucki SMD, Nitrini R, Caramelli P, Bertolucci PHF, Okamoto IH Suggestions for utilization of the mini-mental state examination in Brazil. Arq Neuropsiquiatr. 2003;61(3B):777-81. http://dx.doi. org/10.1590/S0004-282X2003000500014. PMid:14595482.

7. ABEP: Associação Brasileira de Empresas de Pesquisa. Critério de classificação econômica Brasil [Internet]. 2014 [citado em 2020 Jan 1]. Disponível em: http://www.abep.org/criterio-brasil

8. Apolinario D, Braga RC, Magaldi RM, Busse AL, Campora F, Brucki $\mathrm{S}$, et al. Short assessment of health literacy for portuguese-speaking adults. Rev Saude Publica. 2012;46(4):702-11. http://dx.doi.org/10.1590/ S0034-89102012005000047. PMid:22782124.

9. Padovani AR, Moraes DP, Mangili LD, Andrade CRF. Protocolo Fonoaudiológico de Avaliação do Risco para Disfagia (PARD). Rev 
Soc Bras Fonoaudiol. 2007;12(3):199-205. http://dx.doi.org/10.1590/ S1516-80342007000300007.

10. Crary MA, Mann GDC, Groher ME. Initial psychometric assessment of a functional oral intake scale for dysphagia in stroke patients. Arch Phys Med Rehabil. 2005;86(8):1516-20. http://dx.doi.org/10.1016/j. apmr.2004.11.049. PMid:16084801.

11. Silbergleit AK, Schultz L, Jacobson BH, Beardsley T, Johnson AF. The Dysphagia Handicap Index: development and Validation. Dysphagia. 2012;27(1):46-52. http://dx.doi.org/10.1007/s00455-011-9336-2. PMid:21424584.

12. Santos MIPO, Portella MR. Conditions of functional health literacy of an elderly diabetics group. Rev Bras Enferm. 2016;69(1):144-64. http://dx.doi.org/10.1590/0034-7167.2016690121i. PMid:26871229.

13. Moraes KL, Brasil VV, Oliveira GF, Cordeiro JABL, Silva AMTC, Boaventura RP, et al. Functional health literacy and knowledge of renal patients on pre-dialytic treatment. Rev Bras Enferm. 2017;70(1):15562. http://dx.doi.org/10.1590/0034-7167-2015-0169. PMid:28226055.

14. Maragno CAD, Mengue SS, Moraes CG, Rebelo MVD, Guimarães AMM, Pizzol TSD. Test of health Literacy for Portuguese-speaking Adults. Rev Bras Epidemiol. 2019;22:e190025. http://dx.doi. org/10.1590/1980-549720190025. PMid:30942331.

15. IBGE: Instituto Brasileiro de Geografia e Estatística. Censo demográfico [Internet], 2010 [citado em 2020 Maio 25]. Disponível em: http:// www.censo2010. ibge.gov.br/sinopse/index.php?dados $=26 \& u f=31$

16. Romero SS, Scortegagna HM, Doring M. Functional health literacy level and behavior in the health of the elderly. Texto Contexto Enferm. 2018;27(4):e5230017. http://dx.doi.org/10.1590/010407072018005230017 .

17. Marques SRL, Lemos SMA. Health literacy and associated factors in adults primary care users. Trab Educ Saúde. 2018;16(2):535-59. http://dx.doi.org/10.1590/1981-7746-sol00109.

18. Borges FM, Silva ARV, Lima LHO, Almeida PC, Vieira NFC, Machado ALG. Health literacy of adults with and without arterial hypertension. Rev Bras Enferm. 2019;72(3):646-86. http://dx.doi.org/10.1590/00347167-2018-0366. PMid:31269128.

19. Botton A, Cúnico SD, Strey MN. Diferenças de Gênero no acesso aos serviços de saúde: problematizações necessárias. Mudanças Psicol Saúde. 2017;25(1):67-72. http://dx.doi.org/10.15603/2176-1019/mud.v25n1p67-72.
20. Aytar A, Tüzün EH, Eker L. Prevalence and related factors of limited health literacy in patients with chronic musculoskeletal diseases. Turk J Physiother Rehabil. 2017;28(2):54-9.

21. Warren M, DeCarlo DK, Dreer LE. Health literacy in older adults with and without low vision. Am J Occup Ther. 2016;70(3):7. http:// dx.doi.org/10.5014/ajot.2016.017400.

22. von Wühlisch FS, Pascoe M. Maximising health literacy and client recall of clinical information: an exploratory study of clients and speech-language pathologists. SAJCD. 2010;57(1):22-32. http:// dx.doi.org/10.4102/sajcd.v57i1.46. PMid:21329263.

23. OMS: Organização Mundial de Saúde. CIF: a Classificação Internacional de Funcionalidade, Incapacidade e Saúde. 1. ed. São Paulo: Edusp; 2015.

24. Borges MGS, Medeiros AM, Lemos SMA. The International Classification of Functioning, Disability and Health for Children and Youth (ICF-CY) and Speech-language Pathology: associations with sociodemographic and health care variables. CoDAS. 2020;32(3):e20190058. http:// dx.doi.org/10.1590/2317-1782/20202019058. PMid:32609224.

25. Luna JS, Monteiro GTR, Koifman RJ, Bergmann A. Classificação Internacional de Funcionalidade na reabilitação profissional: instrumentos de avaliação da incapacidade laboral. Rev Saude Publica. 2020;54:45. http://dx.doi.org/10.11606/s1518-8787.2020054001463. PMid:32491090.

26. Lima JP, Abreu DPG, Bandeira EO, Brum NA, Mello MCVA, Varela VS, et al. Ealth literacy and associated factors in the elderly. Cogitare Enferm. 2019;24:e63964. http://dx.doi.org/10.5380/ce.v24i0.63964.

27. Fleisher J, Bhatia R, Margus C, Pruitt A, Dahodwala N. Health literacy and medication awareness in outpatient neurology. Neurol Clin Pract. 2014;4(1):71-81. http://dx.doi.org/10.1212/01.CPJ.0000436211.73013. ab. PMid:24605271.

28. Santana JF, Toledo Neto JL, Bravo DS, Costa AB. Challenges and potential of health literacy in the context of empowerment: a systematic literature review. Glob Health Promot. 2017;5(2):211-24.

29. Kobayashi LC, Smith SG, O’Conor R, Curtis LM, Park D, von Wagner $\mathrm{C}$, et al. The role of cognitive function in the relationship between age and health literacy: a cross-sectional analysis of older adults in Chicago, USA. BMJ Open. 2015;5(4):e007222. http://dx.doi. org/10.1136/bmjopen-2014-007222. PMid:25908675. 